\title{
Lyapunov functionals for output regulation of exponentially stable semigroups via integral action and application to a hyperbolic systems
}

\author{
A. Terrand-Jeanne, V. Andrieu, V. Dos Santos Martins, C.-Z. Xu
}

\begin{abstract}
The paper deals with output feedback stabilization of exponentially stable systems by integral controllers. We propose appropriate Lyapunov functionals to prove exponential stability of the closed-loop systems. An example of parabolic PDE (partial differential equation) systems and an example of hyperbolic systems are worked out to show how exponentially stabilizing integral controllers are designed. The contribution of the paper is extending the forwarding technique to infinitedimensional systems by elaborating Lyapunov functionals.
\end{abstract}

\section{INTRODUCTION}

The use of integral action to achieve output regulation for infinite dimensional systems has been initiated by $\mathrm{S}$. Pojohlainen in [10]. It has been extended in a series of papers by the same author (see [11] for instance) and some other (see [?]) always considering bounded control operator and following a spectral approach.

In the last two decades, Lyapunov approaches have allowed to consider a large class of boundary control problems (see for instance [2]). In this work our aim is to follow a Lyapunov approach to solve an output regulation problem. The results are separated into two parts.

In a first part, abstract Cauchy problems are considered. It is shown how a Lyapunov functional can be constructed for a linear system in closed loop with an integral controller when some bounds are assumed on the control and measurement operators. This gives an alternative proof to the results of S. Pojohlainen in [10] (and [?]). It allows also to give explicit value to the integral gain that solves the output regulation problem.

In a second part, following the Lyapunov construction procedure, we consider a boundary regulation problem for a class of hyperbolic PDE systems. This result generalizes many others which have been obtained so far in the regulation of PDE hyperbolic systems (see for instance [7], [17], [3], [15], [16], [14]).

The paper is organized as follows. Section II is devoted to the regulation of the measured output for stable abstract Cauchy problems. It is given a general procedure, for an exponentially stable semigroup in open-loop, to construct a Lyapunov functional for the closed loop system obtained with an integral controller. Inspired by this procedure, the case of boundary regulation is considered for a general class of hyperbolic PDE systems in Section III]. The proof of the theorem obtained in the context of hyperbolic systems is given in Section IV

All authors are with Université de Lyon, LAGEP-CNRS, Université Claude Bernard Lyon1, Domaine Universitaire de la Doua, 43 bd du 11 Novembre 1918, 69622 Villeurbanne Cedex, France.
Note that due to space limitation proofs of technical lemmas have been removed and will be reported in a journal version of this work.

Notation: subscripts $t, s, t t, \ldots$ denote the first or second derivative w.r.t. the variable $t$ or $s$. For an integer $n, \mathrm{I}_{\mathrm{d} n}$ is the identity matrix in $\mathbb{R}^{n \times n}$. Given an operator $\mathcal{A}$ over a Hilbert space, $\mathcal{A}^{*}$ denotes the adjoint operator. $\mathcal{D}_{n}$ is the set of diagonal matrix in $\mathbb{R}^{n \times n}$.

\section{General Abstract Cauchy Problems}

\section{A. Problem description}

Let $\mathcal{X}$ be a Hilbert space with scalar product $\langle,\rangle_{\mathcal{X}}$ and $\mathcal{A}: D(\mathcal{A}) \subset \mathcal{X} \rightarrow \mathcal{X}$ be the infinitesimal generator of a $C_{0^{-}}$ semigroup denoted $t \mapsto e^{\mathcal{A} t}$. Let $\mathcal{B}$ and $\mathcal{C}$ be respectively linear operators from $\mathbb{R}^{m}$ to $\mathcal{X}$ and from $\mathcal{X}$ to $\mathbb{R}^{m}$.

In this section, we consider the controlled Cauchy problem with output $\Sigma(\mathcal{A}, \mathcal{B}, \mathcal{C})$ in Kalman form, as follows

$$
x_{t}=\mathcal{A} x+\mathcal{B} u+w, y=\mathcal{C} x,
$$

where $w \in \mathcal{X}$ is an unknown constant vector and $u$ is the control input. We consider the following exponential stability property for the operator $\mathcal{A}$.

Assumption 1 (Exponential Stability): The operator $\mathcal{A}$ generates an exponentially stable $C_{0}$-semigroup. In other words, there exist $\nu$ and $k$ both positive constants such that, $\forall x_{0} \in \mathcal{X}$ and $t \in \mathbb{R}_{+}$

$$
\left\|e^{\mathcal{A} t} x_{0}\right\|_{\mathcal{X}} \leq k \exp (-\nu t)\left\|x_{0}\right\|_{\mathcal{X}} .
$$

We are interested in the regulation problem. More precisely we are concerned with the problem of regulation of the output $y$ via the integral control

$$
u=k_{i} K_{i} z, z_{t}=y-y_{r e f},
$$

where $y_{r e f} \in \mathbb{R}^{m}$ is a prescribed reference, $z \in \mathbb{R}^{m}, K_{i} \in$ $\mathbb{R}^{m \times m}$ is a full rank matrix and $k_{i}$ a positive real number.

The control law being dynamical, the state space has been extended. Considering the system $\Sigma(\mathcal{A}, \mathcal{B}, \mathcal{C})$ in closed loop, with integral control law given in (3) the state space is now $\mathcal{X}_{e}=\mathcal{X} \times \mathbb{R}^{m}$ which is a Hilbert space with inner product

$$
\left\langle x_{e a}, x_{e b}\right\rangle_{\mathcal{X}_{e}}=\left\langle x_{a}, x_{b}\right\rangle_{\mathcal{X}}+z_{a}^{\top} z_{b},
$$

where $x_{e a}=\left[\begin{array}{l}x_{a} \\ z_{a}\end{array}\right]$ and $x_{e b}=\left[\begin{array}{l}x_{b} \\ z_{b}\end{array}\right]$. The associated norm is denoted $\|\cdot\|_{\mathcal{X}_{e}}$. Let $\mathcal{A}_{e}: D(\mathcal{A}) \times \mathbb{R}^{m} \rightarrow \mathcal{X}_{e}$ be the extended operator defined as

$$
\mathcal{A}_{e}=\left[\begin{array}{cc}
\mathcal{A} & \mathcal{B} K_{i} k_{i} \\
\mathcal{C} & 0
\end{array}\right]
$$


The regulation problem to solve can be rephrased as the following.

Regulation problem: We wish to find a positive real number $k_{i}$ and a full rank matrix $K_{i}$ such that $\forall\left(w, y_{\text {ref }}\right) \in \mathcal{X} \times \mathbb{R}^{m}$ :

1) The system (1)-(3) is well-posed. In other words, for all $x_{e 0}=\left(x_{0}, z_{0}\right) \in \mathcal{X}_{e}$ there exists a unique (weak) solution denoted $x_{e}(t)=\left[\begin{array}{l}x(t) \\ z(t)\end{array}\right] \in C^{0}\left(\mathbb{R}_{+}, \mathcal{X}_{e}\right)$ defined $\forall t \geq 0$ and initial condition $x_{e}(0)=x_{e 0}$.

2) There exists an equilibrium point denoted $x_{e \infty}=$ $\left[\begin{array}{l}x_{\infty} \\ z_{\infty}\end{array}\right] \in \mathcal{X}_{e}$, depending on $w$ and $y_{\text {ref }}$, which is exponentially stable for the system (1)-(3). In other words, there exist positive real numbers $\nu_{e}$ and $k_{e}$ such that for all $t \geq 0$

$$
\left\|x_{e}(t)-x_{e \infty}\right\|_{\mathcal{X}_{e}} \leq k_{e} \exp ^{-\nu_{e} t}\left\|x_{e 0}-x_{e \infty}\right\|_{\mathcal{X}_{e}} .
$$

3) The output $y$ is regulated toward the reference $y_{r e f}$. More precisely,

$$
\forall x_{e 0}, \lim _{t \rightarrow+\infty}\left|\mathcal{C} x(t)-y_{r e f}\right|=0 .
$$

We know with the work of S. Pohjolainen in [11] (see also [?]) that the following theorem holds.

Theorem 1 ([11], [?]): Under Assumption 1, assume that:

1) the operator $\mathcal{B}$ is bounded;

2) the operator $\mathcal{C}$ is $\mathcal{A}$-bounded, i.e.

$$
|\mathcal{C} x| \leq c\left(\|x\|_{\mathcal{X}}+\|\mathcal{A} x\|_{\mathcal{X}}\right), \forall x \in D(\mathcal{A}),
$$

for some positive real number $c$;

$3)$ the rank condition holds. In other words operators $\mathcal{A}$, $\mathcal{B}$ and $\mathcal{C}$ satisfy

$$
\operatorname{rank}\left\{\mathcal{C} \mathcal{A}^{-1} \mathcal{B}\right\}=m
$$

then there exists a positive real number $k_{i}^{*}$ and a $m \times m$ matrix $K_{i}$, such that for all $0<k_{i} \leq k_{i}^{*}$ the operator $\mathcal{A}_{e}$ given in 4 is the generator of an exponentially stable $C_{0^{-}}$ semigroup in the extended state space $\mathcal{X}_{e}$. More precisely, the system (1) in closed loop with (3) is well-posed and the equilibrium is exponentially stable. Moreover, for all $w$ and $y_{\text {ref }}$, equation (5) holds (i.e the regulation is achieved).

On the other hand, if one wants to address nonlinear abstract Cauchy problems or unbounded operators, we may need to follow a Lyapunov approach. For instance in the context of boundary control, a Lyapunov functional approach has allowed to tackle feedback stabilization of a large class of PDEs (see for instance [2] or [5]).

It is well known (see for instance [8, Theorem 8.1.3]) that exponential stability of the operator $\mathcal{A}$ is equivalent to existence of a bounded positive and self adjoint operator $\mathcal{P}$ in $\mathfrak{L}(X)$ such that

$$
\langle\mathcal{A} x, \mathcal{P} x\rangle_{\mathcal{X}}+\langle\mathcal{P} x, \mathcal{A} x\rangle_{\mathcal{X}} \leq-\omega\|x\|_{\mathcal{X}}^{2}, \forall x \in D(\mathcal{A}),
$$

where $\omega$ is a positive real number. We assume that this Lyapunov operator $\mathcal{P}$ is given. The first question that we intend to solve is the following: Knowing the Lyapunov operator $\mathcal{P}$, is it possible to construct a Lyapunov operator $\mathcal{P}_{e}$ associated to the extended operator $\mathcal{A}_{e}$ ?

To answer this question, we first give a construction based on a well-known technique in the nonlinear finite dimensional control community named the forwarding (see for instance [9], [13] or more recently [4], or [1]).

\section{B. A Lyapunov approach for regulation}

Inspired by the forwarding techniques, the following result can be obtained.

Theorem 2 (Forwarding Lyapunov functional): Assume that all assumptions of Theorem 1 are satisfied and let $\mathcal{P}$ in $\mathfrak{L}(X)$ be a self adjoint operator such that 7 holds. Then there exist a bounded operator $\mathcal{M}: \mathcal{X} \rightarrow \mathbb{R}^{m}$ and positive real numbers $p$ and $k_{i}^{*}$, such that for all $0<k_{i} \leq k_{i}^{*}$, there exists $\omega_{e}>0$ such that the operator

$$
\mathcal{P}_{e}=\left[\begin{array}{cc}
\mathcal{P}+p \mathcal{M}^{*} \mathcal{M} & -p \mathcal{M}^{*} \\
-p \mathcal{M} & p \mathrm{I}_{\mathrm{d}}
\end{array}\right]
$$

satisfies $\forall x_{e}=(x, z) \in D(\mathcal{A}) \times \mathbb{R}^{m}$

$\left\langle\mathcal{A}_{e} x_{e}, \mathcal{P}_{e} x_{e}\right\rangle_{\mathcal{X}_{e}}+\left\langle\mathcal{P}_{e} x_{e}, \mathcal{A}_{e} x_{e}\right\rangle_{\mathcal{X}_{e}} \leq-\omega_{e}\left(\|x\|_{\mathcal{X}}^{2}+|z|^{2}\right)$.

Proof: Let $\mathcal{M}: \mathcal{X} \rightarrow \mathbb{R}^{m}$ be defined by $\mathcal{M}=\mathcal{C} \mathcal{A}^{-1}$. Note that we have,

$$
|\mathcal{M} x|=\left|\mathcal{C} \mathcal{A}^{-1} x\right| \leq c\left(\left\|\mathcal{A}^{-1} x\right\|_{\mathcal{X}}+\|x\|_{\mathcal{X}}\right) \leq \tilde{c}\|x\|_{\mathcal{X}},
$$

where $\tilde{c}$ is a positive real number. First inequality comes from the fact that $\mathcal{C}$ is $\mathcal{A}$-bounded while the second one express the well-know property establishing that the inverse of an operator generating a $C_{0}$-semigroups stable is bounded. Hence, $\mathcal{M}$ is a bounded linear operator. Moreover, $\mathcal{M}$ satisfies the following equation

$$
\mathcal{M A} x=\mathcal{C} x, \forall x \in D(\mathcal{A}) .
$$

Let $K_{i}=\left(\mathcal{C A}^{-1} \mathcal{B}\right)^{-1}$ which exists due to the third assumption of Theorem 1 . Note that,

$$
\left\langle x_{e}, \mathcal{P}_{e} x_{e}\right\rangle_{\mathcal{X}_{e}}=\langle x, \mathcal{P} x\rangle_{\mathcal{X}}+p(z-\mathcal{M} x)^{\top}(z-\mathcal{M} x) .
$$

This candidate Lyapunov functional is similar to the one given in [4, Equation (34)]. It is selected following a forwarding approach.

Moreover, we have

$$
\begin{aligned}
& \left\langle\mathcal{A}_{e} x_{e}, \mathcal{P}_{e} x_{e}\right\rangle_{\mathcal{X}_{e}}+\left\langle\mathcal{P}_{e} x_{e}, \mathcal{A}_{e} x_{e}\right\rangle_{\mathcal{X}_{e}}= \\
& \langle\mathcal{A} x, \mathcal{P} x\rangle_{\mathcal{X}}+\langle\mathcal{P} x, \mathcal{A} x\rangle_{\mathcal{X}} \\
& +2 p(z-\mathcal{M} x)^{\top}(\mathcal{C} x-\mathcal{M} \mathcal{A} x)+k_{i}\left\langle x, \mathcal{P} \mathcal{B} K_{i} z\right\rangle_{\mathcal{X}} \\
& +k_{i}\left\langle\mathcal{P} \mathcal{B} K_{i} z, x\right\rangle_{\mathcal{X}}-2 p(z-\mathcal{M} x)^{\top} \mathcal{M B} K_{i} k_{i} z .
\end{aligned}
$$

Let $\left\|\mathcal{P B} K_{i}\right\|_{\mathcal{X}}^{2}=L$ which is well defined due to the boundedness assumption on $\mathcal{B}$. Employing equation 110 and $\mathcal{M B} K_{i}=\mathrm{I}_{\mathrm{d}}$, the former inequality becomes

$$
\begin{aligned}
& \left\langle\mathcal{A}_{e} x_{e}, \mathcal{P}_{e} x_{e}\right\rangle_{\mathcal{X}_{e}}+\left\langle\mathcal{P}_{e} x_{e}, \mathcal{A}_{e} x_{e}\right\rangle_{\mathcal{X}_{e}}= \\
& \langle\mathcal{A} x, \mathcal{P} x\rangle_{\mathcal{X}}+\langle\mathcal{P} x, \mathcal{A} x\rangle_{\mathcal{X}}+k_{i}\left\langle x, \mathcal{P} \mathcal{B} K_{i} z\right\rangle_{\mathcal{X}} \\
& \quad+k_{i}\left\langle\mathcal{P} \mathcal{B} K_{i} z, x\right\rangle_{\mathcal{X}}-2 p(z-\mathcal{M} x)^{\top} k_{i} z .
\end{aligned}
$$


Given $a, b$ positive constants, the following inequalities hold

$$
\begin{aligned}
\left\langle x, \mathcal{P B} K_{i} z\right\rangle_{\mathcal{X}} & \leq \frac{1}{2 a}\|x\|_{\mathcal{X}}^{2}+\frac{a L}{2}|z|^{2}, \\
z^{\top} \mathcal{M} x & \leq \frac{1}{2 b}\|x\|^{2}+\frac{b\|\mathcal{M}\|^{2}}{2}|z|^{2},
\end{aligned}
$$

it yields

$$
\begin{aligned}
\left\langle\mathcal{A}_{e} x_{e}, \mathcal{P}_{e} x_{e}\right\rangle_{\mathcal{X}_{e}} & +\left\langle\mathcal{P}_{e} x_{e}, \mathcal{A}_{e} x_{e}\right\rangle_{\mathcal{X}_{e}} \\
\leq & \left(-\omega+\frac{k_{i}}{a}+\frac{p k_{i}}{b}\right)\|x\|_{\mathcal{X}}^{2} \\
& +k_{i}\left(p\left(-2+b\|\mathcal{M}\|^{2}\right)+a L\right)|z|^{2} .
\end{aligned}
$$

We pick $b$ sufficiently small such that

$$
-2+b\|\mathcal{M}\|^{2}<0 .
$$

In a second step, we select $a$ sufficiently small and $p$ sufficiently large such that

$$
p\left(-2+b\|\mathcal{M}\|^{2}\right)+a L<0 .
$$

Finally, picking $k_{i}^{*}$ sufficiently small such that

$$
-\omega+\frac{k_{i}^{*}}{a}+\frac{p k_{i}^{*}}{b}<0
$$

the result is obtained with

$$
\omega_{e}=\min \left\{\omega-\frac{k_{i}}{a}-\frac{p k_{i}}{b}, p\left(2-b\|\mathcal{M}\|^{2}\right)-a L\right\} .
$$

\section{Discussion on the result}

A direct interest of the Lyapunov approach given in Theorem 2, is that it allows to give an explicit value for $k_{i}^{*}$ which appears in Theorem 1

Corollary 1 (Explicit integral gain): Given a system $\Sigma(\mathcal{A}, \mathcal{B}, \mathcal{C})$ satisfying the assumptions of the Theorem 1. points 1), 2), and 3) of Theorem 1 hold with $K_{i}=\left(\mathcal{C A}^{-1} \mathcal{B}\right)^{-1}$ and

$$
k_{i}^{*}=\frac{\nu}{\left\|\mathcal{C} \mathcal{A}^{-1}\right\| k^{2}\left\|\mathcal{B}\left(\mathcal{C A}^{-1} \mathcal{B}\right)^{-1}\right\|} .
$$

An interesting question would now to know in which aspect this value may be optimal.

\section{Illustration on a parabolic systems}

Consider the problem of heating a bar of length $L=10$ with both endpoints at temperature zero. We control the heat flow in and out around the points $s=2,5$, and 7 and measure the temperature at points 3,6 , and 8 . The problem is to find an integral controller such that the measurements at $s=3,6$, and 8 are regulated to (for instance) 1,3 , and 2 , respectively. Thus the control system is governed by the following PDE

$$
\begin{gathered}
\phi_{t}(s, t)=\phi_{s s}(s, t)+\mathbb{1}_{\left[\frac{3}{2}, \frac{5}{2}\right]}(s) u_{1}(t)+\mathbb{1}_{\left[\frac{9}{2}, \frac{11}{2}\right]}(s) u_{2}(t) \\
\quad+\mathbb{1}_{\left[\frac{13}{2}, \frac{15}{2}\right]}(s) u_{3}(t), \quad(s, t) \in(0,10) \times(0, \infty)
\end{gathered}
$$

where $\phi:[0,+\infty) \times[0,10] \rightarrow \mathbb{R}$ with boundary conditions

$$
\phi(0, t)=\phi(10, t)=0
$$

$$
\phi(s, 0)=\phi_{0}(s),
$$

where $\mathbb{1}_{[a, b]}:[0,10] \rightarrow \mathbb{R}$ denotes the characteristic function on the interval $[a, b]$, i.e.,

$$
\mathbb{1}_{[a, b]}(s)= \begin{cases}1 & \forall s \in[a, b], \\ 0 & \forall s \notin[a, b] .\end{cases}
$$

The output and the reference are given as

$$
y(t)=\left[\begin{array}{l}
\phi(t, 3) \\
\phi(t, 6) \\
\phi(t, 8)
\end{array}\right], y_{\text {ref }}=\left[\begin{array}{l}
1 \\
3 \\
2
\end{array}\right] .
$$

Let the state space be the Hilbert space $\mathcal{X}=$ $L^{2}((0,10), \mathbb{R})$ with usual inner product, and let the input space and the output space be equal to $\mathbb{R}^{3}$. Clearly, from 21, we get the semigroup generator $\mathcal{A}: D(\mathcal{A}) \rightarrow \mathcal{X}$, the input operator $\mathcal{B}: \mathbb{R}^{3} \rightarrow \mathcal{X}$ and the output operator $\mathcal{C}: D(\mathcal{A}) \rightarrow \mathbb{R}^{3}$ as follows:

$$
D(\mathcal{A})=\left\{\varphi \in H^{2}(0,10) \mid \varphi(0)=\varphi(10)=0\right\},
$$

and

$$
\begin{gathered}
\mathcal{A} \varphi=\varphi_{s s} \forall \varphi \in D(\mathcal{A}), \\
\mathcal{B} u=\mathbb{1}_{\left[\frac{3}{2}, \frac{5}{2}\right]} u_{1}+\mathbb{1}_{\left[\frac{9}{2}, \frac{11}{2}\right]} u_{2}+\mathbb{1}_{\left[\frac{13}{2}, \frac{15}{2}\right]} u_{3},
\end{gathered}
$$

and

$$
C \varphi=\left[\begin{array}{lll}
\varphi(3) & \varphi(6) & \varphi(8)
\end{array}\right]^{T}[1
$$

Moreover, note that with Sobolev embedding, an integration by part and by completing the square, we have for all $\varphi$ in $D(\mathcal{A})$

$$
\begin{aligned}
\sup _{s \in(0,10)}|\varphi(s)| & \leq c \int_{0}^{10} \varphi(s)^{2} d s+c \int_{0}^{10} \varphi_{s}(s)^{2} d s \\
& \leq c\|\varphi\|_{\mathcal{X}}+c \int_{0}^{10}\left|\varphi(s) \varphi_{s s}(s)\right| d s \\
& \leq \frac{3}{2} c\|\varphi\|_{\mathcal{X}}+\frac{1}{2} c\left\|\varphi_{s s}\right\|_{\mathcal{X}} .
\end{aligned}
$$

Hence $\mathcal{C}$ is $\mathcal{A}$-bounded.

Moreover, by direct computation we find that

$$
\mathcal{C A}^{-1} \mathcal{B}=\frac{-1}{10}\left[\begin{array}{ccc}
14 & 15 & 9 \\
8 & 20 & 18 \\
4 & 10 & 14
\end{array}\right]
$$

It is easy to see that the above matrix is regular. Consequently all Assumptions of Theorem 1 hold. With Corollary 1 it is possible to compute explicitly the integral controller gain. By direct computation we have for all $\varphi$ in $\mathcal{X}$

$$
\mathcal{C A}^{-1} \varphi=\left[\begin{array}{l}
\frac{3}{10} \int_{0}^{10}(s-10) \varphi(s) d s+\int_{0}^{3}(3-s) \varphi(s) d s \\
\frac{3}{5} \int_{0}^{10}(s-10) \varphi(s) d s+\int_{0}^{6}(6-s) \varphi(s) d s \\
\frac{4}{5} \int_{0}^{10}(s-10) \varphi(s) d s+\int_{0}^{8}(8-s) \varphi(s) d s
\end{array}\right],
$$

which gives $\left\|\mathcal{C} \mathcal{A}^{-1}\right\| \leq 6.2466$. We have

$$
K_{i}=\left[\begin{array}{ccc}
-1.250 & 1.500 & -1.125 \\
0.500 & -2.000 & 2.250 \\
0 & 1.000 & -2.000
\end{array}\right] \text {. }
$$

${ }^{1} X^{T}$ denotes the transposed state 
For the open-loop system, consider the Lyapunov operator $\mathcal{P}=\mathrm{I}_{\mathrm{d}}$. Then the growth rate may be taken as $\omega=\frac{\pi^{2}}{50}$. It is easy to see that $\left\|K_{i}\right\|=4.2433$, and $\|\mathcal{B}\| \leq \sqrt{3}$. Putting together the numerical values into the formula (19) allows to estimate the tuning parameter

$$
k_{i}^{*}=\frac{\omega}{2\left\|\mathcal{B} K_{i}\right\|\left\|\mathcal{C} \mathcal{A}^{-1}\right\|} \approx 2.1498 * 10^{-3} .
$$

With Corollary 1, the integral controller (3) with $0<k_{i}<$ $2.1498 * 10^{-3}$ stabilizes exponentially the equilibrium along solutions of the closed-loop system and drives asymptotically the measured temperatures to the reference values for any initial condition.

\section{CASE OF BOUNDARY REGULATION}

\section{A. Case of unbounded operators}

It is interesting to remark that a possible way to remove the boundedness assumption on the operators $\mathcal{C}$ and $\mathcal{B}$ can be made as follow.

Corollary 2 (Regulation with unbounded operators):

Assume Assumption 1 holds and let $\mathcal{P}$ in $\mathfrak{L}(X)$ be a self adjoint operator such that 7 holds. Assume that:

1) the operator $\mathcal{C} \mathcal{A}^{-1}$ is bounded;

2) the rank condition holds, that is to say, the operators $\mathcal{A}$, $\mathcal{B}$ and $\mathcal{C}$ satisfy equation $\left(6\right.$. Let $K_{i}=\left(\mathcal{C} \mathcal{A}^{-1} \mathcal{B}\right)^{-1}$.

3) there exists a positive real numbers $L$ and $a$ such that

$$
\left\langle x, \mathcal{P B} K_{i} z\right\rangle \leq \frac{1}{2 a}\|x\|_{\mathcal{X}}^{2}+\frac{a L}{2}|z|^{2}
$$

4) the operator $\mathcal{A}_{e}$ given in (4) is the generator of $C_{0^{-}}$ semigroup in the extended state space $X_{e}$.

Then there exists a positive real number $k_{i}^{*}$ such that for all $0<k_{i} \leq k_{i}^{*}$ the operator $\mathcal{A}_{e}$ is the generator of an exponentially stable $C_{0}$-semigroup in the extended state space $\mathcal{X}_{e}$. In other words, the equilibrium of the system (1) in closed loop with the control law (3), is exponentially stable.

In the context of hyperbolic PDE systems with boundary control, this is typically the kind of results that can be used in order to achieve regulation. In the following section we adapt this framework to hyperbolic PDE systems. Note however that since the system is not given in a Kalman form, it is not a direct application of Corollary 2. Nevertheless, the approach follows exactly the same line.

\section{B. System description}

To illustrate the former abstract theory, we consider the case of hyperbolic partial differential equations as studied in [6]. More precisely, the system is given by a one dimensional $n \times n$ hyperbolic system

$$
\phi_{t}=\Lambda \phi_{x}, x \in(0,1), t \in[0,+\infty),
$$

where $\phi:[0,+\infty) \times[0,1] \rightarrow \mathbb{R}^{n}$

$$
\begin{aligned}
\Lambda & =\operatorname{diag}\left\{\lambda_{1}, \ldots, \lambda_{n}\right\} \\
\lambda_{i} & >0 \forall i \in\{1, \ldots, \ell\} \\
\lambda_{i} & <0 \forall i \in\{\ell+1, \ldots, n\} \\
\lambda_{i} & \neq \lambda_{j} \forall(i, j) \in\{1, \ldots, n\}^{2} \text { such that } i \neq j,
\end{aligned}
$$

with the initial condition $\phi(0, x)=\phi_{0}(x)$ for $x$ in $[0,1]$ where $\phi_{0}:[0,1] \rightarrow \mathbb{R}^{n}$ and with the boundary conditions

$$
\begin{aligned}
& {\left[\begin{array}{l}
\phi_{+}(t, 0) \\
\phi_{-}(t, 1)
\end{array}\right]=K\left[\begin{array}{l}
\phi_{+}(t, 1) \\
\phi_{-}(t, 0)
\end{array}\right]+B u_{b}(t)+w_{b}} \\
& \quad=\left[\begin{array}{ll}
K_{11} & K_{12} \\
K_{21} & K_{22}
\end{array}\right]\left[\begin{array}{l}
\phi_{+}(t, 1) \\
\phi_{-}(t, 0)
\end{array}\right]+\left[\begin{array}{l}
B_{1} \\
B_{2}
\end{array}\right] u_{b}(t)+w_{b}
\end{aligned}
$$

where $\phi=\left[\begin{array}{l}\phi_{+} \\ \phi_{-}\end{array}\right]$with $\phi^{+}$in $\mathbb{R}^{\ell}, \phi^{-}$in $\mathbb{R}^{n-\ell}$ and where $w_{b}$ in $\mathbb{R}^{n}$ is an unknown disturbance, $u(t)$ is a control input taking values in $\mathbb{R}^{m}$ and $K$ and $B$ are matrices of appropriate dimensions.

The output to be regulated to a prescribed value denoted by $y_{r e f}$, is given as a disturbed linear combination of the boundary conditions. Namely, the outputs to regulate are in $\mathbb{R}^{m}$ given as

$$
y(t)=L_{1}\left[\begin{array}{l}
\phi_{+}(t, 0) \\
\phi_{-}(t, 1)
\end{array}\right]+L_{2}\left[\begin{array}{l}
\phi_{+}(t, 1) \\
\phi_{-}(t, 0)
\end{array}\right]+w_{y},
$$

where $L_{1}$ and $L_{2}$ are two matrices in $\mathbb{R}^{m \times n}$ and $w_{y}$ is an unknown disturbance in $\mathbb{R}^{m}$. We wish to find a positive real number $k_{i}$ and a full rank matrix $K_{i}$ such that

$$
u(t)=k_{i} K_{i} z(t), z_{t}(t)=y(t)-y_{\text {ref }}, z(0)=z_{0}
$$

where $z(t)$ takes value in $\mathbb{R}^{m}$ and $z_{0} \in \mathbb{R}^{m}$ solves the regulation problem $\forall y_{\text {ref }} \in \mathbb{R}^{m}$.

The state space denoted by $\mathcal{X}_{e}$ of the system 22)-23 in closed loop with the control law 26 is the Hilbert space defined as:

$$
\mathcal{X}_{e}=\left(L^{2}(0,1), \mathbb{R}^{n}\right) \times \mathbb{R}^{m},
$$

equipped with the norm defined for $x_{e}=(\phi, z)$ in $\mathcal{X}_{e}$ as:

$$
\|v\|_{\mathcal{X}_{e}}=\|\phi\|_{L^{2}\left((0,1), \mathbb{R}^{m}\right)}+|z| .
$$

We introduce also a smoother state space defined as:

$$
\mathcal{X}_{e 1}=\left(H^{1}(0,1), \mathbb{R}^{n}\right) \times \mathbb{R}^{m} .
$$

\section{Output regulation result}

In this section, we give a set of sufficient conditions allowing to solve the regulation problem as described in the introduction. Our approach follows what we have done in the former section.

Assumption 2 (Input-to-State Exponential Stability):

There exist a $C^{1}$ function $P:[0,1] \rightarrow \mathcal{D}_{n}$, a real number $\mu>0$ and a positive definite matrix $S$ in $\mathbb{R}^{n \times n}$ such that

$$
\begin{array}{r}
-P_{s}(s) \Lambda \leq-\mu P(s), \\
\underline{p} \mathrm{I}_{\mathrm{d} n} \leq P(s) \leq \bar{p} \mathrm{I}_{\mathrm{d} n}, \forall s \in[0,1],
\end{array}
$$

and

$$
\begin{aligned}
& {\left[\begin{array}{cc}
\mathrm{I}_{\mathrm{d} \ell} & K_{21}^{\top} \\
0 & K_{22}^{\top}
\end{array}\right] P(1) \Lambda\left[\begin{array}{cc}
\mathrm{I}_{\mathrm{d} \ell} & 0 \\
K_{21} & K_{22}
\end{array}\right]} \\
& \quad-\left[\begin{array}{lc}
K_{11}^{\top} & 0 \\
K_{12}^{\top} & \mathrm{I}_{\mathrm{d} n-\ell}
\end{array}\right] P(0) \Lambda\left[\begin{array}{cc}
K_{11} & K_{12} \\
0 & \mathrm{I}_{\mathrm{d} n-\ell}
\end{array}\right] \leq-S .
\end{aligned}
$$

As it will be seen in the following section, this assumption is a sufficient condition for exponential stability of the 
equilibrium of the open loop system. It can be found in [2] in the case in which $S$ may be semi-definite positive. The positive definiteness of $S$ is fundamental to get an input-tostate stability (ISS) property of the open loop system with respect to the disturbances on the boundary. More general results are given in [12].

The second assumption is related to the rank condition.

Assumption 3 (Rank condition): The matrix

$$
T=\left(L_{1}+L_{2}\right)\left(\mathrm{I}_{\mathrm{d} n}-K\right)^{-1} B
$$

is well defined and full rank.

With these assumptions, the following result may be obtained.

Theorem 3 (Regulation for hyperbolic PDE systems):

Assume that Assumptions 2 and 3 are satisfied then with $K_{i}=T^{-1}$ there exists $k_{i}^{*}>0$ such that for all $0<k_{i}<k_{i}^{*}$ the output regulation is obtained. More precisely, for all $\left(w_{b}, w_{y}, y_{\text {ref }}\right)$ in $\mathbb{R}^{p} \times \mathbb{R}^{m} \times \mathbb{R}^{m}$, the following holds.

1) For all $\left(\phi_{0}, z_{0}\right)$ in $\mathcal{X}_{e}$ (resp. $\left.\mathcal{X}_{1 e}\right)$ which satisfies the boundary conditions (23) (resp. the $C^{1}$ compatibility condition), there exists a unique weak solution to (22)-23)-(26) that we denote $v$ and which belongs to $C^{0}\left([0,+\infty) ; \mathcal{X}_{e}\right)$ (Respectively, strong solution in:

$$
\left.C^{0}\left([0,+\infty) ; \mathcal{X}_{e 1}\right) \cap C^{1}\left([0,+\infty) ; \mathcal{X}_{e}\right)\right) .
$$

2) There exists an equilibrium state denoted $v_{\infty}$ in $\mathcal{X}_{e}$ which is globally exponentially stable in $\mathcal{X}_{e}$ for system (22)-23)-26. More precisely, we have for all $t \geq 0$ :

$$
\left\|v(t)-v_{\infty}\right\|_{\mathcal{X}_{e}} \leq k \exp (-\nu t)\left\|v_{0}-v_{\infty}\right\|_{\mathcal{X}_{e}} .
$$

3) Moreover, if $v_{0}$ satisfies the $C^{1}$-compatibility condition and is in $\mathcal{X}_{1 e}$, the regulation is achieved, i.e.

$$
\lim _{t \rightarrow+\infty}\left|y(t)-y_{\text {ref }}\right|=0 .
$$

The next section is devoted to the proof of this result.

\section{Proof of TheOREM 3}

To prove the main result, we demonstrate that under some conditions, the desired regulation is obtained provided there exists a Lyapunov functional for system (22)-23)-26. Then, it only remains to explicitly build the Lyapunov functional to end the proof.

\section{A. Stabilization implies regulation}

In this first subsection, we explicitly give the equilibrium state of the system (22)-23)-(26). We show also that if we assume that $k_{i}$ and $K_{i}$ are selected such that this equilibrium point is exponentially stable along the closed loop, then the regulation is achieved.

1) Definition of the equilibrium: The first step of the study is to exhibit equilibrium denoted $\phi_{\infty}, z_{\infty}$ of the disturbed hyperbolic PDE in closed loop with the boundary integral control (i.e. system (22)-(26).

We have the following proposition.

Proposition 1: Assumption 3 is a sufficient condition for the existence of an equilibrium of the system 22)-23 - 26 for all $y_{r e f}$ and $w_{b}$. Moreover, if Assumption 3 holds then point 1) of Theorem 3 holds.

The proof of this result has been removed due to space limitation and will be available in the journal version of this paper.

We can introduce $\tilde{\phi}(x, t)=\phi(x, t)-\phi_{\infty}$ and $\tilde{z}(t)=$ $z(t)-z_{\infty}$. It can be checked that $\tilde{\phi}, \tilde{z}$ satisfies the following system:

$$
\begin{aligned}
\tilde{\phi}_{t} & =\Lambda \tilde{\phi}_{x}, x \in(0,1), \\
z_{t} & =L_{1}\left[\begin{array}{l}
\tilde{\phi}_{+}(t, 0) \\
\tilde{\phi}_{-}(t, 1)
\end{array}\right]+L_{2}\left[\begin{array}{l}
\tilde{\phi}_{+}(t, 1) \\
\tilde{\phi}_{-}(t, 0)
\end{array}\right], t \in[0,+\infty),
\end{aligned}
$$

with the boundary conditions

$$
\begin{aligned}
{\left[\begin{array}{c}
\tilde{\phi}_{+}(t, 0) \\
\tilde{\phi}_{-}(t, 1)
\end{array}\right] } & =K\left[\begin{array}{l}
\tilde{\phi}_{+}(t, 1) \\
\tilde{\phi}_{-}(t, 0)
\end{array}\right]+B u(t), \\
u(t) & =k_{i} K_{i} \tilde{z}(t) .
\end{aligned}
$$

2) Sufficient conditions for Regulation: In the following, we show that the regulation problem can be rephrased as a stabilization of the equilibrium state introduced previously.

Proposition 2: Assume Assumption 3holds and that there exist a functional $W: \mathcal{X}_{e} \rightarrow \mathbb{R}_{+}$, and positive real numbers $\omega$ and $L_{w}$ such that:

$$
\frac{\left\|v_{\infty}-v\right\|_{\mathcal{X}_{e}}^{2}}{L_{w}} \leqslant W(v) \leqslant L_{w}\left\|v_{\infty}-v\right\|_{\mathcal{X}_{e}}^{2} .
$$

Assume moreover that for all $v_{0}$ in $\mathcal{X}_{e}$ and all $t_{0}$ in $\mathbb{R}_{+}$such that the solution $v$ of system 22)-23)-26 initiated from $v_{0}$ is $C^{1}$ at $t=t_{0}$, we have:

$$
W_{t}(t) \leqslant-\omega W(t)
$$

where with a slight abuse of notation $W(t)=W(v(t))$. Then points 1), 2) and 3) of Theorem 3 hold.

Sketch of the proof: Point 1) is directly obtained from Proposition 1 . The proof of point 2) is by now standard. The last point is obtained employing Sobolev embedding and showing the convergence of trajectories toward the equilibrium in the supremum norm.

With this proposition in hand, to prove the Theorem 3, it is sufficient to construct a Lyapunov functional $W$ which satisfies 237-238) along $C^{1}$ solutions of 22)-23-26 or equivalently along $C^{1}$ solutions of (34)-(35). This is considered in the next section following the route of Section II-B

\section{B. Lyapunov functional construction}

B.1) Open loop ISS: Inspired by the Lyapunov functional construction introduced in [6] (see also [2]), we know that typical Lyapunov functionals allowing to exhibit stability property for this type of hyperbolic PDE are given as functional $V: L^{2}\left((0,1), \mathbb{R}^{n}\right) \rightarrow \mathbb{R}_{+}$defined as

$$
V(\varphi)=\int_{0}^{1} \varphi(s)^{\top} P(s) \varphi(s) d s
$$

where $P:(0,1) \rightarrow \mathbb{R}^{n \times n}$ is a $C^{1}$ function. Typically in [6], these functions are taken as exponentials. With a slight abuse of notation, we write $V(t)=V(\tilde{\phi}(\cdot, t))$ and we denote 
by $\dot{V}(t)$ the time derivative of the Lyapunov functional along solutions which are $C^{1}$ in time. In our context, with assumption 2, it yields the following proposition.

Proposition 3: If Assumption 2 holds, there exists a positive real number $c$ such that for every solution $\phi$ of 22)-23 initiated from $\left(\tilde{\phi}_{0}, \tilde{z}_{0}\right)$ in $\mathcal{X}_{e}$ which satisfies 35

$$
\dot{V}(t) \leq-2 \mu V(t)+c|u(t)|^{2} .
$$

The proof of this proposition has been removed due to space limitation.

B.2) Forwarding approach to deal with the integral part: Following the route of Section II-B, a Lyapunov functional is designed from $V$ adding some terms to take into account the state of the integral controller. Let the operator $\mathcal{M}$ : $L^{1}\left((0,1) ; \mathbb{R}^{n}\right) \rightarrow \mathbb{R}^{m}$ be given as

$$
\begin{aligned}
\mathcal{M} \varphi & =\int_{0}^{1} M \Lambda^{-1} \varphi(s) d s \\
& =\int_{0}^{1} M_{+} \Lambda_{+}^{-1} \varphi_{+}(s)+M_{-} \Lambda_{-}^{-1} \varphi_{-}(s) d s
\end{aligned}
$$

where $\varphi=\left[\begin{array}{c}\varphi_{+} \\ \varphi_{-}\end{array}\right], M=\left[\begin{array}{ll}M_{+} & M_{-}\end{array}\right]$and where $M_{+}$and $M_{-}$are two matrices respectively in $\mathbb{R}^{m \times \ell}$ and in $\mathbb{R}^{m \times(n-\ell)}$ that have to be selected. Following the Lyapunov functional construction in Theorem 2, we consider the candidate Lyapunov functional $W: L^{2}\left((0,1) ; \mathbb{R}^{n}\right) \times \mathbb{R}^{m}$ given as

$$
W(\varphi, z)=V(\varphi)+p(z-\mathcal{M} \varphi)^{\top}(z-\mathcal{M} \varphi) .
$$

Note that there exists a positive real number $L_{w}$ such that for all $(\varphi, z)$ in $\mathcal{X}_{e}$

$$
\frac{1}{L_{w}}\left(\|\varphi\|_{\mathcal{X}}^{2}+|z|^{2}\right) \leq W(\varphi, z) \leq L_{w}\left(\|\varphi\|_{\mathcal{X}}^{2}+|z|^{2}\right) .
$$

In the following theorem, it is shown that by selecting properly $K_{i}, k_{i}, M$ and $p$, this function is indeed a Lyapunov functional for the closed loop system. Again, with a slight abuse of notation, we write $W(t)=W(\tilde{\phi}(\cdot, t), \tilde{z}(t))$ and we denote by $\dot{W}(t)$ the time derivative of the Lyapunov functional along solutions which are $C^{1}$ in time.

Proposition 4: Assume that Assumptions 2 and 3 hold. Then there exists a matrix $K_{i}$ and $k_{i}^{*}$ such that for all $k_{i}<$ $k_{i}^{*}$, along $C^{1}$ solution of the system (34)-35- 36

$$
\dot{W}(t) \leqslant-\omega_{2} W(t), \forall t \in \mathbb{R}_{+} .
$$

The proof of this proposition is removed due to space limitation.

With this proposition, the proof of Theorem 3 is completed.

\section{CONCLUSION}

In the last three decades, the regulation problem has been studied for different classes of distributed parameter systems. Most of existing results follow a semigroup approach and the perturbation theory for linear operator. In this paper we have shown that is was also possible to construct Lyapunov functionals to address the regulation problem in the case in which is used an integral action. This framework allows to explicitly give an integral gain. Moreover, it is no more necessary to impose boundedness of control or measurement operators to guarantee the regulation. This is applied to PDE hyperbolic systems and this allows to generalize many available results in this field.

\section{REFERENCES}

[1] Daniele Astolfi and Laurent Praly. Integral action in output feedback for multi-input multi-output nonlinear systems. IEEE Transactions on Automatic Control, 62(4):1559-1574, 2017.

[2] Georges Bastin and Jean-Michel Coron. Stability and boundary stabilization of 1-d hyperbolic systems, volume 88. Springer, 2016.

[3] Georges Bastin, Jean-Michel Coron, and Simona Oana Tamasoiu. Stability of linear density-flow hyperbolic systems under pi boundary control. Automatica, 53:37-42, 2015.

[4] S. Benachour, V. Andrieu, L. Praly, and H. Hammouri. Forwarding design with prescribed local behavior. IEEE Transactions on Automatic Control, 58(12):3011-3023, Dec 2013.

[5] Jean-Michel Coron. Control and nonlinearity. Number 136. American Mathematical Soc., 2007.

[6] Jean-Michel Coron, Georges Bastin, and Brigitte d'Andréa Novel. Dissipative boundary conditions for one-dimensional nonlinear hyperbolic systems. SIAM Journal on Control and Optimization, 47(3):14601498, 2008.

[7] V Dos Santos, Georges Bastin, J-M Coron, and Brigitte dAndréa Novel. Boundary control with integral action for hyperbolic systems of conservation laws: Stability and experiments. Automatica, 44(5):13101318, 2008.

[8] Birgit Jacob and Hans J Zwart. Linear port-Hamiltonian systems on infinite-dimensional spaces, volume 223. Springer, 2012.

[9] F. Mazenc and L. Praly. Adding integrations, saturated controls, and stabilization for feedforward systems. IEEE Transactions on Automatic Control, 41(11):1559-1578, 1996.

[10] Seppo Pohjolainen. Robust multivariable pi-controller for infinite dimensional systems. IEEE Transactions on Automatic Control, 27(1):17-30, 1982.

[11] Seppo Pohjolainen. Robust controller for systems with exponentially stable strongly continuous semigroups. Journal of mathematical analysis and applications, 111(2):622-636, 1985.

[12] Christophe Prieur and Frédéric Mazenc. Iss-lyapunov functions for time-varying hyperbolic systems of balance laws. Mathematics of Control, Signals, and Systems, 24(1-2):111-134, 2012.

[13] R. Sepulchre, M. Jankovic, and P.V. Kokotovic. Integrator forwarding: a new recursive nonlinear robust design. Automatica, 33(5):979-984, 1997.

[14] Alexandre Terrand-Jeanne, Valérie Dos-Santos Martins, and Vincent Andrieu. Regulation of the downside angular velocity of a drilling string with a p-i controller. In Proceedings of European Control Conference, 2018.

[15] N.-T. Trinh, V. Andrieu, and C.-Z. Xu. Design of integral controllers for nonlinear systems governed by scalar hyperbolic partial differential equations. IEEE Transactions on Automatic Control, 2017.

[16] N.-T. Trinh, V. Andrieu, and C.-Z. Xu. Stability and output regulation for a cascaded network of $2 \times 2$ hyperbolic systems with pi control. Automatica, submitted.

[17] Cheng-Zhong Xu and Gauthier Sallet. Multivariable boundary pi control and regulation of a fluid flow system. Mathematical Control and Related Fields, 4(4):501-520, 2014. 